\title{
DRIFT COMPRESSION OF SPACE CHARGE DOMINATED BEAMS*
}

\author{
M. J. L. de Hoon ${ }^{\dagger}$, E. P. Lee, LBNL, Berkeley, CA 94720, USA \\ J. J. Barnard, LLNL, Livermore, CA 94550, USA
}

\begin{abstract}
Heavy ion driven inertial fusion drivers and experiments use space-charge dominated beams that require longitudinal bunch compression to increase the power delivered on target. In these scenarios, a drift compression section is designed such that the longitudinal compression stagnates as a result of the space charge repulsion of the beam. Special attention is given to keeping all parts of the beam matched, which is not trivial since the rate of current increase varies along the beam.

A conceptual drift compression section was designed for a space-charge dominated beam. It will be shown that all parts of the beam can be kept approximately matched.
\end{abstract}

\section{INTRODUCTION}

In current designs of heavy-ion drivers for inertial fusion, the beams are compressed longitudinally after the accelerator stage to increase the power deposited on target. Longitudinal compression can be achieved by imposing a head-to-tail velocity tilt on the beam. This tilt has to be carefully tailored, such that it is removed by the longitudinal space-charge repulsion by the time the beam reaches the end of the drift compression section.

The longitudinal electric field generated by the beam is shielded by the surrounding conducting pipe to a degree depending on the beam and pipe radii. Therefore, the transverse beam dynamics affects the longitudinal dynamics. Conversely, the transverse dynamics of the beam depends on the current and hence on the longitudinal compression or expansion that the beam has undergone. So the longitudinal and transverse dynamics of a space-charge dominated beam are coupled. In order to design a drift compression section, in which the current changes continuously and the average beam radius varies in some specified way, we need to resort to iteration over small longitudinal distances. On each iteration, small changes are made to the lattice properties, until the transverse and longitudinal dynamics of the beam have converged.

\section{DRIFT COMPRESSION DESIGN}

In this paper, an example of a drift compression section is shown for the Integrated Research Experiment (IRE), which is the next major step envisioned in the Heavy Ion Inertial Fusion program [1]. At the end of the IRE accelerator, the $\mathrm{K}^{+}$beam has a kinetic energy of $200 \mathrm{MeV}$.

\footnotetext{
* Work performed under the auspices of the U.S. Department of Energy under University of California contract W-7405-ENG-48 at LLNL, and University of California contract DE-AC-76SF00098 at LBNL.

† mdehoon@lbl.gov
}

No further acceleration takes place in the drift compression section. Each beam has a charge of $4.6875 \mu \mathrm{C}$, with a pulse duration of $336 \mathrm{~ns}$ at the end of the accelerator. In the example described below, the pulse duration is reduced to about $20 \mathrm{~ns}$ after drift compression, corresponding to an average beam current of $234.375 \mathrm{~A}$ for a flat current profile.

Typically, the transverse beam size needs to be large in the final focus section in order to be able to focus the beam onto a small spot on target, whereas the beam size is kept small for economic reasons in the accelerator. This requires a controlled transverse expansion of the beam during drift compression. We therefore specify how we would like the average beam radius to vary as a function of $z$. For the calculations presented here, we chose a smooth increase given by

$$
\begin{aligned}
\bar{a}(z)= & a_{\mathrm{acc}}+\frac{1}{2}\left(a_{\mathrm{ff}}-a_{\mathrm{acc}}\right) . \\
& {\left[1+\tanh \left(\cot \left(\pi \frac{L_{\mathrm{dc}}-z}{L_{\mathrm{inc}}}\right)\right)\right], }
\end{aligned}
$$

for $z>L_{\mathrm{dc}}-L_{\mathrm{inc}}$, and $\bar{a}(z)=a_{\mathrm{acc}}$ for $z<L_{\mathrm{dc}}-L_{\mathrm{inc}}$. Here, $a_{\mathrm{acc}}$ is the average beam radius at the end of the accelerator, as determined by the current IRE design, $a_{\mathrm{ff}}$ is the average beam radius at the beginning of the final focus section, $z$ is the distance from the beginning of the drift compression section, $L_{\mathrm{dc}}$ is the total length of the drift compression system, and $L_{\text {inc }}$ is the distance over which we want the beam radius to increase from $a_{\text {acc }}$ to $a_{\mathrm{ff}}$. Other choices, such as a simple linear falloff, may cause mismatches to occur near discontinuities in $\bar{a}(z)$ or $\bar{a}^{\prime}(z)$.

For the calculations presented here, the Hermes code was used [2], which treats the beam as a cold fluid longitudinally and employs the envelope equation transversely. The longitudinal electric field is calculated by approximating the beam as being cylindrically symmetric, and depositing the beam charge onto an RZ grid. The longitudinal electric field is then found using WARP's RZ field solver [3]. For the large compression factors considered here, the $g$-factor model proved to be insufficiently accurate for detailed numerical calculations [2].

Designing a drift compression section consists of one backward and one forward Hermes run. In the backward run, the drift compression section is set up starting from a desired final beam pulse at the end of drift compression. We choose a beam duration we would like to achieve there, and we find the corresponding final current. We assume the current profile to be a flat-top, with $25 \%$ parabolic fall-offs on each side. At final focus, the occupancy $\eta$ was chosen to be $65 \%$, which is near the practically achievable limit. For the design presented here, the occupancy was held constant in the drift compression section, although that is not 
a necessary design requirement. The undepressed tune was chosen to be $72^{\circ}$, which is low enough for the beam to be stable. The beam radius at the entrance of the final focus section was chosen to be $6 \mathrm{~cm}$, compared to $1.76 \mathrm{~cm}$ at the end of the IRE accelerator. These numerical values allow us to calculate the perveance at final focus and the lattice half period that we would need for a transport lattice, as well as the magnetic field gradient in the quadrupoles of a transport lattice to achieve a tune of $72^{\circ}$.

Next, a large number of lattice half periods of this transport lattice is set up. We initialize a Hermes beam in the last couple of half periods, making sure the entire beam is covered by the lattice. The beam is set up such that its tail is at the center of a drift space. The beam is then matched transversely to the transport lattice.

The beam is simulated backward in time, from the end of the drift compression to the beginning. In order to set up the lattice, we need to iterate over each lattice half period. We first save the parameters of the beam at the beginning of an iteration step. We then run Hermes backwards in time, each time step being negative, until the center of the beam reaches the center of the preceding lattice half period. Since the beam has expanded longitudinally, the current at the beam center has decreased. Therefore, the lattice half period and focusing force of this half period do not exactly correspond to the actual current at the beam center. For the next iteration step, we adjust the half period length and the focusing force by first evaluating from equation (1) the desired average beam radius $\bar{a}$ at the center of the lattice half period. We then calculate the perveance $Q$ from the current at the beam center that was found from the previous iteration step, and evaluate the approximate relation [4]

$$
Q\left(\frac{2 L}{\bar{a}}\right)^{2} \approx 2\left(\cos \sigma-\cos \sigma_{0}\right),
$$

in which $\sigma_{0}=72^{\circ}$ and $\sigma$ are the undepressed and depressed tune respectively, to find the lattice half period $L$ for the next iteration step. Since the depressed tune $\sigma$ is small for space-charge dominated beams, usually we can set $\cos \sigma=1$ in this equation. Finally, we use the approximate relation [4]

$$
\cos \sigma_{0} \approx 1-\frac{1}{2}\left(\eta k L^{2}\right)^{2}\left(1-\frac{2}{3} \eta\right),
$$

to find the focusing strength $k$ of the quadrupoles. The aperture is chosen based on the heuristic

$$
\text { aperture }=1.25 \times \text { maximum beam radius }+5 \mathrm{~mm} \text {, }
$$

allowing a $5 \mathrm{~mm}$ clearance for misalignments [5]. Setting up the aperture correctly is crucial, since the magnitude of the longitudinal electric field depends on the shielding effect by the aperture.

Once we know the properties of the lattice half period needed for the next iteration step, we adjust the previous half period, and all other half periods to the left of it, such that its length and quadrupole strength correspond to the current we found. The Hermes beam is then reloaded from its saved values, and Hermes is again run through this half period. Once the difference between the calculated half period length and the actual half period length is smaller than a given error limit, our solution has converged and the procedure is repeated for the next lattice half period. For the calculations presented here, an error limit of $1 \times 10^{-9}$ was chosen. The iteration converges quickly, typically taking about six steps for each half period. This procedure is continued until the current at the beam center has reduced to the level at the end of the accelerator. The quadrupoles that have not been used are then removed from the simulation.

\section{EXAMPLE}

An example drift compression section was designed using $L_{\text {inc }}=150 \mathrm{~m}$ in equation (1). Figure 1 shows the beam radius at the center of the beam as well as near the beam ends, together with the aperture. Although the procedure outlined above keeps the center of the beam approximately matched, the ends of the beam become mismatched. Mismatches will occur if locally or globally the current increases rapidly compared to a betatron period, preventing the beam from adjusting itself adiabatically. Whereas the current at the beam center increases from $100 \mathrm{~A}$ to $200 \mathrm{~A}$ in $21.45 \mathrm{~m}$, the current near the ends of the beam increases from $16 \mathrm{~A}$ to $32 \mathrm{~A}$ over only $8.24 \mathrm{~m}$, which is less than the betatron period of $9.66 \mathrm{~m}$. This results in a severe mismatch near the ends of the beam, as shown in figure 1 . The mismatch will be even worse if the final pulse duration or the distance $L_{\text {inc }}$ in equation (1) is reduced.

Note that the mismatch is produced at the end of the drift compression section, where the beam current changes

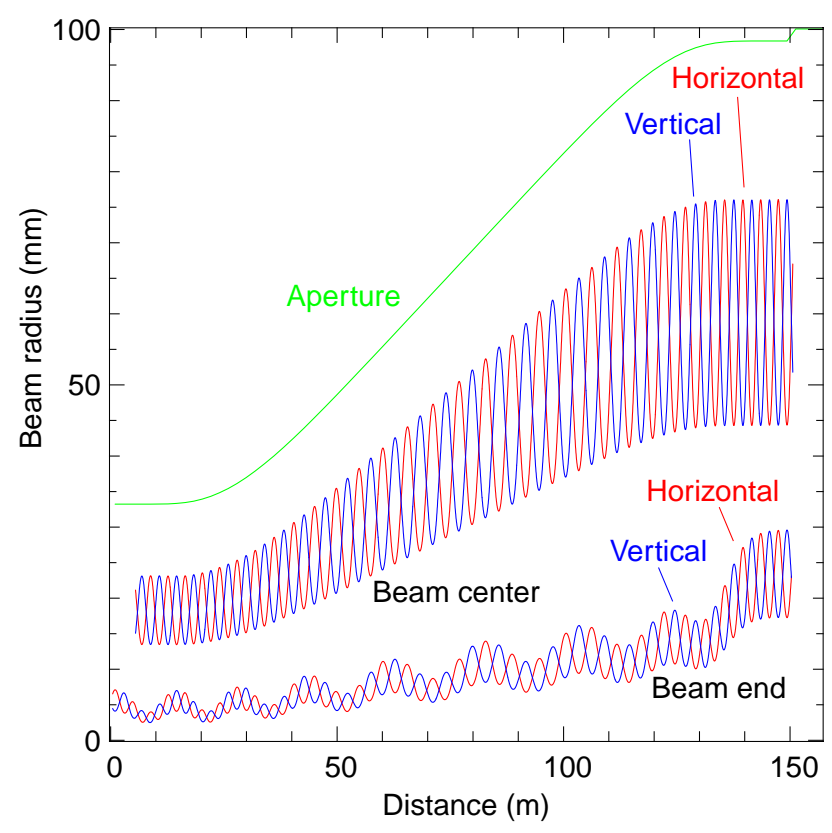

Figure 1: The transverse beam envelope if the beam is not rematched at the beginning of drift compression. 


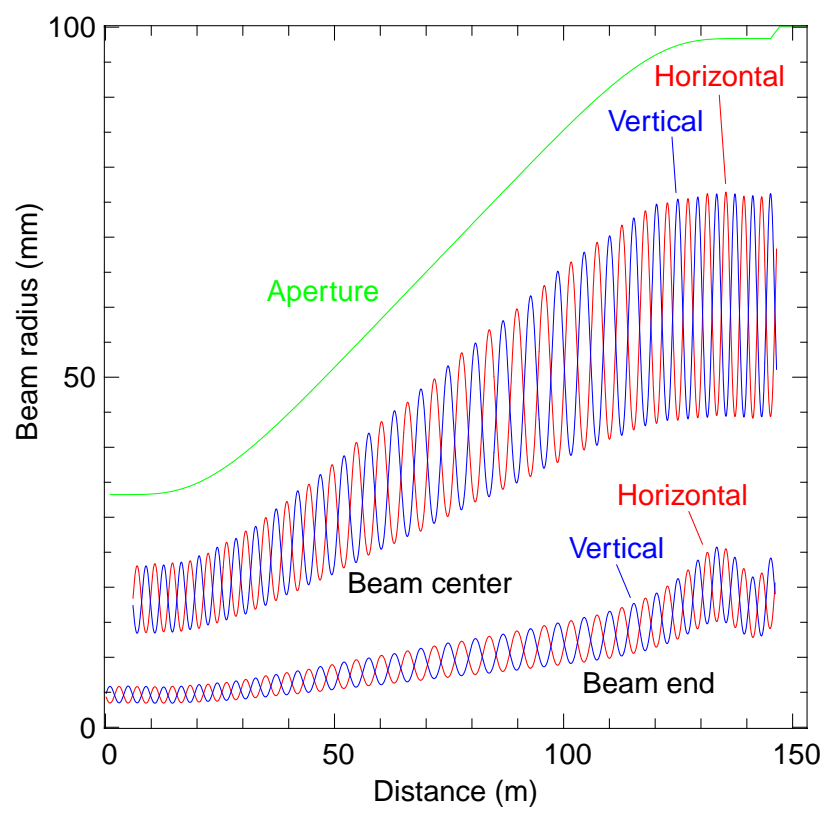

Figure 2: The transverse beam envelope if the beam is rematched at the beginning of drift compression.

most rapidly. In the backward run, the mismatch then persists up to the beginning of the drift compression section. These mismatches are undesirable, since they may result in an emittance increase as well as halo formation. To minimize the distance of the drift compression over which mismatches occur, we should therefore rematch the beam once the backward run reaches the beginning of the drift compression section. The simulation is then run forward from the beginning of the drift compression to the end. In this forward run of the rematched beam, some mismatch will now occur near the end of drift compression. Since this mismatch will be formed fairly late, it will not affect the beam severely. Figure 2 shows the beam radius if we rematch at the beginning of the drift compression. The beam remains matched for most of the drift compression, although a small mismatch develops near the end.

Rematching the beam does not significantly affect the longitudinal dynamics, as indicated by the RMS difference between the final and the originally specified current profile being only $0.65 \%$. Figure 3 shows the current profile at the end of drift compression, if the beam is rematched at the beginning of drift compression.

Figure 4 shows the initial velocity tilt that was found from our calculation, together with the initial current profile. Since the velocity profile is smooth, we believe that such a velocity profile is achievable in practice.

\section{REFERENCES}

[1] J. J. Barnard et al., "Planning for an Integrated Research Experiment", 13th International Symposium on Heavy Ion Inertial Fusion, San Diego, California, 2000.

[2] M. J. L. de Hoon, "Drift Compression and Final Focus Sys-

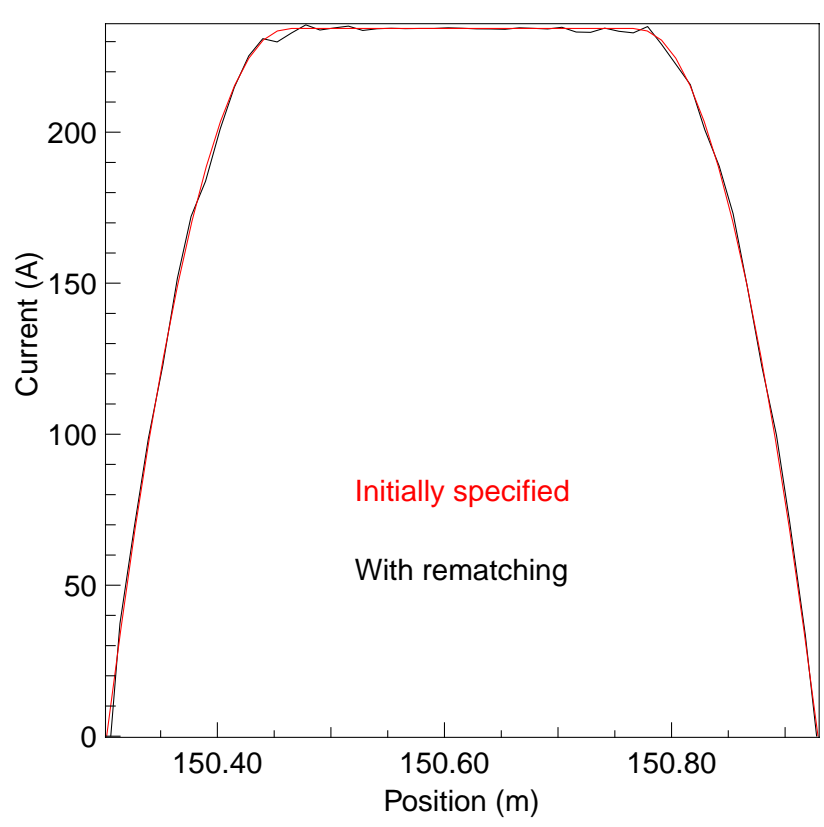

Figure 3: The current profile as a function of position at the end of drift compression, if the beam is rematched at the beginning of drift compression.

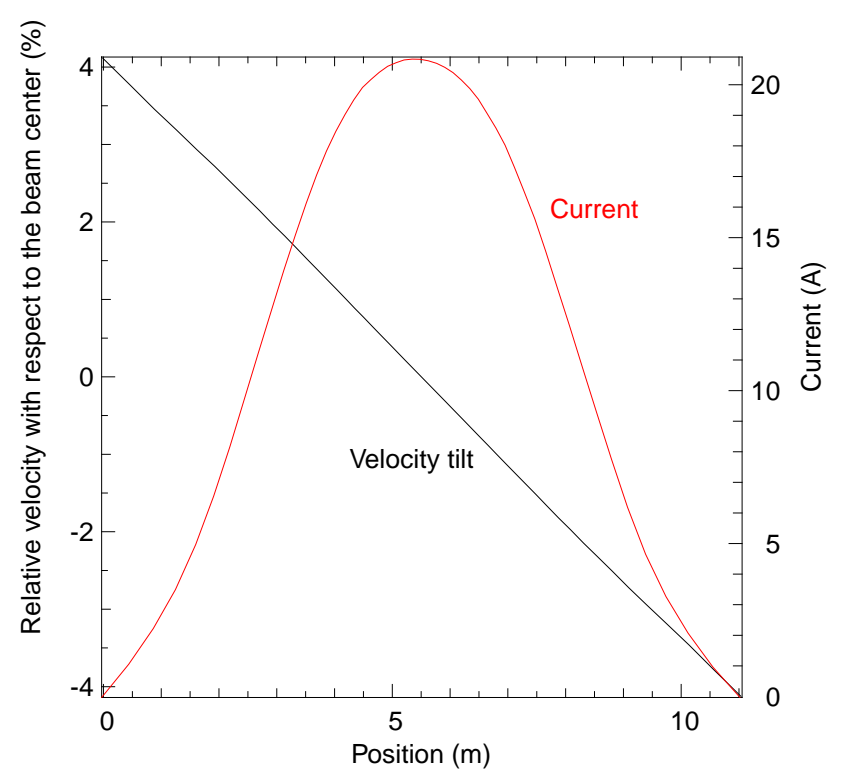

Figure 4: The initial velocity tilt.

tems for Heavy Ion Inertial Fusion”, Ph.D. thesis, University of California, Berkeley, 2001.

[3] D. A. Callahan-Miller, "Simulations of Longitudinal Beam Dynamics of Space-Charge Dominated Beams for Heavy Ion Fusion”, Ph.D. thesis, University of California, Davis, 1994.

[4] Edward P. Lee, "The Beam Envelope Equation - Systematic Solution for a Periodic Quadrupole Lattice with Space Charge", Particle Accelerators, 52, 115-132, 1996.

[5] Andy Faltens and Ed Lee, "Beam Clearances - Revision", HIFAR Note 430, Lawrence Berkeley National Laboratory, Accelerator and Fusion Research Division, 1995. 